\title{
FAST FELDKAMP ALGORITHM FOR CONE-BEAM COMPUTER TOMOGRAPHY
}

\author{
Shu Xiao, Yoram Bresler, and David C. Munson, Jr. \\ Department of Electrical and Computer Engineering and \\ Coordinated Science Laboratory \\ University of Illinois at Urbana-Champaign \\ 1308 W. Main St., Urbana, IL 61801 \\ Email: \{s-xiao@ifp.uiuc.edu,ybresler@uiuc.edu,d-munson@ifp.uiuc.edu\}
}

\begin{abstract}
We propose a new fast Feldkamp algorithm for 3-D cone beam tomography with a circular source trajectory. The algorithm is an extension of our recent fast native 2-D fan-beam reconstruction algorithm. It is based on a recursive hierarchical decomposition of the cone-beam backprojection operation into successively smaller sub-volumes. The algorithm reduces the computational complexity of the reconstruction from $O\left(N^{4}\right)$ to $O\left(N^{3} \log N\right)$. Simulations demonstrate the efficiency of our algorithm, with 7-fold speedup for a $128 \times 128 \times 128$ image. image. Speedups will be much greater for images of more typical size encountered in medicine.
\end{abstract}

\section{INTRODUCTION}

Cone-beam tomography is a three-dimensional extension of twodimensional fan-beam tomography. Rays diverging as a cone from the source illuminate the object, and data corresponding to line integrals along these rays is recorded on a planar or cylindrical detector surface. Such cone-beam projections are collected for a multitude of source positions along a source orbit. The main advantage of cone-beam acquisition is the reduction of data collection time. This is particularly important for real-time imaging of moving structures, such as the beating heart, or contrast agent flow through the body. With the progress in planar detector technology, it is expected that next generation scanners will adopt the cone-beam geometry.

Unfortunately, the computational complexity of the key backprojection step in cone-beam reconstruction algorithms is $O\left(N^{4}\right)$, making them prohibitively slow (or expensive, with parallel hardware implementations) in most applications. In this paper we propose a fast method for cone-beam backprojection, that reduces this cost to $O\left(N^{3} \log N\right)$, yielding multifold speedup in practice.

We focus on the popular single circle source orbit geometry, in which the source moves around the object on a circular orbit. This acquisition geometry can not provide complete data [1] and the reconstructed images are therefore prone to inherent artifacts, in particular away from the source plane. None the less, this geometry is often the most practical, and the Feldkamp, or FDK algorithm [2] developed for it is the reconstruction method most often used in practice. Like all conventional 3D cone-beam reconstruction algorithms, the standard Feldkamp algorithm has an unfavorable $O\left(N^{4}\right)$ computational complexity.

This research was partially supported by DARPA through the U.S. Army Research Office under Contract 38310-PH DAAG55-98-1-0039.
Previous attempts to accelerate the Feldkamp algorithm for cone-beam reconstruction include Turbell's fast algorithms [3,4], which perform a fast backprojection on cone-beam data after rebinning to parallel-beam projections, using the links concept [5]. They therefore suffer from the drawbacks of rebinning - an additional computation cost to perform sufficiently accurate interpolation. The reported speedup over the conventional Feldkamp algorithm [2] for these algorithms is modest - about $30 \%$ for a $256 \times$ $256 \times 128$ object, rising to 7 -fold speedup for a $1024 \times 1024 \times 512$ 3-D image.

The new fast Feldkamp algorithm proposed in this paper is derived from our recent fast fan-beam backprojection algorithm [6]. An instance of the divide-and-conquer principle, the algorithm is based on a decomposition of the cone-beam backprojection operation into smaller sub-volumes. Using the key property that smaller images require fewer projections for reconstruction, the projections are decimated by a factor of two as the image volume is decomposed. Applied recursively, the algorithm requires $O\left(N^{3} \log N\right)$ computations instead of the original $O\left(N^{4}\right)$. Computer simulations demonstrate that for a $128 \times 128 \times 128$ image, our algorithm achieves about a 7 -fold speedup over the original algorithm with little visual quality degradation. This yields a projected 40 -fold speedup for a $1024 \times 1024 \times L$ image, thus showing greater promise in practice than other fast Feldkamp algorithms.

\section{CONE-BEAM RECONSTRUCTION}

We only discuss the case of planar equispaced detectors, where the detectors are evenly spaced on a planar surface. The other common case of detectors equiangularly spaced on a cylindrical surface can be derived in a similar manner.

Figure 1 shows the cone-beam geometry with a single circular orbit and planar detector. The source orbit lies on a circle $\vec{s}(\theta)=$ $[D \cos (\theta), D \sin (\theta), 0]$ of radius $D$ centered at the origin $O$, in the $x, y$ plane. The detector plane, with coordinates $\left(t_{1}, t_{2}\right)$, is assumed, without loss of generality, to contain the $z$ axis (which coincides with the $t_{2}$ axis), and be perpendicular to the source-tocenter line $\overline{S O}$.

The cone-beam projection $(\mathcal{P} f)\left(\theta, t_{1}, t_{2}\right)$ of the object $f$ at source orbit position $\theta$ and detector position $\left(t_{1}, t_{2}\right)$ is the line integral along the source ray parametrized by $\left(\theta, t_{1}, t_{2}\right)$. For particular $\vec{t}$ and $\theta$, the value of $(\mathcal{P} f)(\theta, \vec{t})$ is a sample of the 3D x-ray transform of $f$.

Projections are acquired at $P$ discrete source positions $\theta_{p}=$ $p \Delta \theta, p=0, \ldots P-1$ with uniform spacing $\Delta \theta=\left(\theta_{\max }-\right.$ 


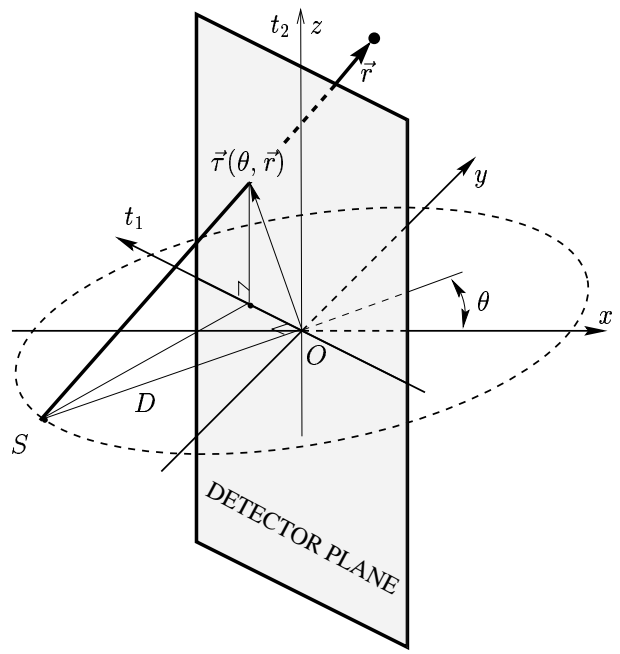

Fig. 1. Cone-beam tomography with circular source orbit and planar equispaced detector.

$\left.\theta_{\text {min }}\right) / P$. We call $(\mathcal{P} f)\left(\theta_{p}, \cdot\right)$ (for all values of $\vec{t}$ ) a projection at source position $\theta_{p}$. The detector plane is usually sampled on a uniform rectangular grid, with possibly different intervals $T_{1}$ and $T_{2}$ on the $t_{1}$ and $t_{2}$ axes.

Feldkamp reconstruction of cone-beam data can be formulated as a weighted filtered backprojection [2]. First, the cone-beam projections are individually weighted and ramp filtered, producing the modified cone-beam projections $g(p, \vec{t})$, corresponding to source positions $\theta_{p}$. For the sake of brevity, we refer to $g(p, \cdot)$ just as "cone-beam projection". Next, the 3D image is reconstructed by a weighted backprojection.

To define the backprojection operation, let $\vec{r}=[x, y, z]^{T}$ denote position in the $3 \mathrm{D}$ image (i.e., object), and let $\vec{\tau}(\theta, \vec{r})=$ $\left[\tau_{1}(\theta, \vec{r}), \tau_{2}(\theta, \vec{r})\right]^{T}$ denote the $t_{1}, t_{2}$ position of the intersection with the detector plane of the source ray passing through point $\vec{r}$ and with source angle $\theta$ (see Fig. 1). Then

$$
\begin{aligned}
\tau_{1}(\theta, \vec{r}) & =\frac{D(y \cos \theta-x \sin \theta)}{D+x \cos \theta+y \sin \theta} \\
\tau_{2}(\theta, \vec{r}) & =\frac{D z}{D+x \cos \theta+y \sin \theta} .
\end{aligned}
$$

The weighted backprojection is then

$$
f(\vec{r})=\sum_{p=0}^{P-1} W(p \Delta \theta, \vec{r}) g[p, \vec{\tau}(p \Delta \theta, \vec{r})] \Delta \theta,
$$

where $W(\theta, \vec{r})$ is an appropriate weight function. This discrete backprojection formula approximates the integral expression for the backprojection with projections measured for all $\theta$. Note that $g(p, \vec{t})$ is also sampled in $\vec{t}$. Hence, interpolation of $g$ in $\vec{t}$ is required to implement the backprojection, because $\vec{\tau}(p \Delta \theta, \vec{r})$ does not usually correspond to an available sample position. The backprojection formula (3) is identical to the 2-D fan-beam case, except that in the latter $\tau$ is scalar, and $\vec{r}$ two dimensional.

The computational cost of 3D cone-beam backprojection for an $N \times N \times N$ image with $P$ projections is $c N^{3} P$, because the contributions of $P$ projections have to be accumulated in (3) for each of the $N^{3}$ image voxels. The constant $c$ depends on implementation details such as complexity of the interpolation. In contrast, the computational cost of weighting and ramp filtering is only $O\left(P N^{2} \log N\right)$ when the convolution is performed using FFTs. Therefore, the cost of backprojection dominates the cost of conventional cone-beam reconstruction, which has cost $O\left(P N^{3}\right)$, or $O\left(N^{4}\right)$, when, as is often the case, $P=O(N)$. The situation is similar in $2 \mathrm{D}$ fan-beam reconstruction, where the complexities of the filtering and backprojection steps are $O\left(N^{2} \log N\right)$ and $O\left(N^{3}\right)$, respectively.

\section{FAST FELDKAMP ALGORITHM}

We first review the fast native fan-beam backprojection algorithm [6]. Its underlying idea is to decompose the $N \times N$ image into smaller subimages and to reconstruct each subimage individually.

Consider the backprojection operation for a sub-image $f^{\prime}(\vec{r})$ of $f$. Let $f^{\prime}=\mathcal{K}_{M}[\vec{\delta}] f$ be a sub-image of $f$ of size $M \times M$, centered at $\vec{\delta}$. The backprojection onto subimage $f^{\prime}$ using $Q$ projections at source angles $p \Delta \theta, p=0 \ldots Q-1$ is given by

$$
f^{\prime}=\mathcal{K}_{M}[\vec{\delta}] f=\mathcal{B}_{M, P}[\vec{\delta}] \hat{\mathcal{K}}_{M}[\vec{\delta}] g
$$

where $\mathcal{B}_{M, Q}[\vec{\delta}]$ is the associated backprojection operator, and $\hat{\mathcal{K}}_{M}[\vec{\delta}]$ is the operator that, for each rotation angle, truncates $g(p, \cdot)$ to the support of the projection of the support of the subimage $f^{\prime}$. That is, the backprojection onto $f^{\prime}$ can be obtained by a backprojection of size $(M, Q)$ of the appropriately truncated projections.

The fast backprojection algorithm uses the decomposition idea with the following additional property. For fixed image resolution (bandwidth), the number of projections needed to reconstruct an image centered at the origin $O$ is proportional to the size of the image [7]. Therefore, if $P$ projections are needed to reconstruct an $N \times N$ image, then $P^{\prime}=P / 2$ projections suffice to reconstruct a $N / 2 \times N / 2$ origin-centered image to the same resolution. The fast backprojection algorithm is therefore based on reconstruction of a subimage $f^{\prime}=\mathcal{K}_{M}[\vec{\delta}] f$ from a reduced number of projections.

The actual reduction of the number of projections used to reconstruct a subimage must account for the fact that subimages are not origin-centered. Therefore, truncated subimage projections $\hat{\mathcal{K}}_{M}[\vec{\delta}] g$ are first radially shifted so that their center aligns with the projection of the origin, then the projections are angularly decimated by a factor $L$ by angular filtering followed by subsampling, and then the projections are radially shifted back so their center again aligns with the projection of the center $\vec{\delta}$ of the subimage. This operation is represented by the operator $\mathcal{O}[L, M, \vec{\delta}]$ which is a composition of the truncation, shift and angular decimation operations.

With these definitions, the exact formula for backprojection onto subimage $f^{\prime}$ is replaced by the approximation

$$
f^{\prime}=\mathcal{K}_{M}[\vec{\delta}] f=\mathcal{B}_{M, P / L}[\vec{\delta}] \mathcal{O}[L, M, \vec{\delta}] g
$$

where $\mathcal{B}_{M, P / L}$ is a backprojection onto an $M \times M$ subimage using $P / L$ projections. This leads to an approximate decomposition of the backprojection operation for a partitioned image, which for $L=2$ and $M=N / 2$ has the form 


$$
f=\mathcal{B}_{N, P}[\overrightarrow{0}] g=\sum_{j=1}^{4} \mathcal{B}_{N / 2, P / 2}\left[\vec{\delta}_{j}\right] \mathcal{O}\left[2, N / 2, \vec{\delta}_{j}\right] g
$$

Applying the decomposition recursively, the total cost of the fast fan-beam reconstruction algorithm becomes $O\left(N^{2} \log N\right)$ [6].

Now consider the 3-D cone-beam backprojection. Figure 2 illustrates the sampling scheme. For a given source position, line integrals are measured at uniformly spaced $z$ intervals, such as $z_{1}$, $z_{2}$. The detectors at a fixed $z$ position form a line parallel to the $x-y$ plane and perpendicular to the $z$ axis. Therefore, all detector lines passing through the same $z$ position from all source postions form a plane parallel to the $x-y$ plane, e.g., planes $p_{1}$ and $p_{2}$ in Fig. 2. We refer to these planes as projection planes.

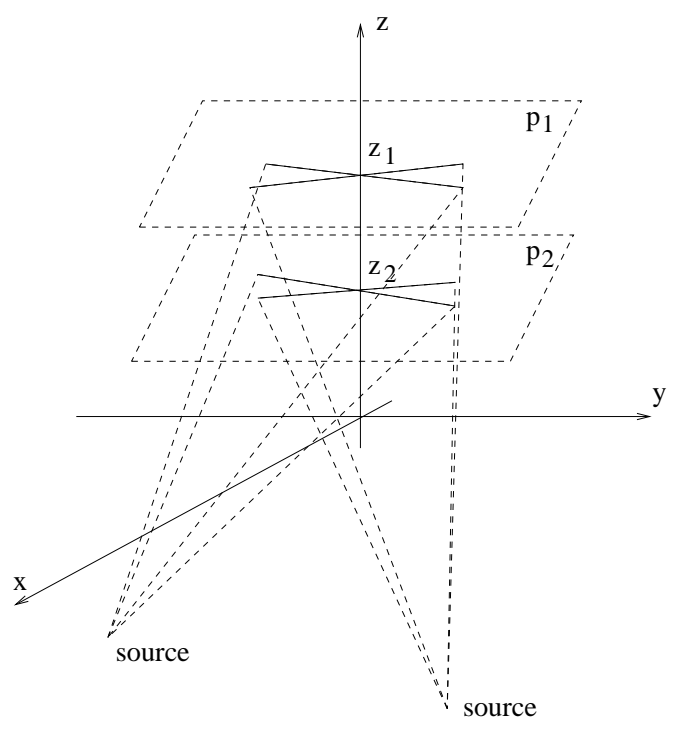

Fig. 2. Cone-beam geometry. Planes $p_{1}$ and $p_{2}$ contain x-ray projections for all source angles at detector positions $z=z_{1}$ and $z=z_{2}$.

For $z=0$, the projection plane is the $x-y$ plane and the data collected from all source angles are the exact fan-beam projections of the object on the $x-y$-plane. Therefore, the 2-D image on the $x-y$-plane can be reconstructed using the fast fan-beam algorithm. We desire a similar fast reconstruction algorithm for other slices of the object at $z \neq 0$. The Feldkamp algorithm performs the weighted backprojection for each pixel in a way that is very similar to the fan-beam backprojection; hence, we propose a fast Feldkamp algorithm for 3-D cone-beam reconstruction as follows.

In our algorithm, each projection plane parallel to the $x-y$ plane is treated as a group. The data on the projection plane are viewed as the fan-beam projections of that plane, so that the whole data set is treated as stacked fan-beam projections of different slices of the 3-D object. Therefore, for each slice, we can perform the same decompostion on the projection plane data as that for fan-beam projections. Considering the decomposition simultaneously on all slices, the original volume is decomposed to four "pillars" as in Fig. 3. For a $a \times b \times c$ object, each sub-volume has size $a / 2 \times b / 2 \times c$. For each sub-volume, the projections are shifted, angularly decimated, and shifted back slice-by-slice, as in the fast fan-beam algorithm [6]. The overall effect is that for each sub-volume, the total number of cone-beam projections (source positions) for reconstruction is decreased by half.

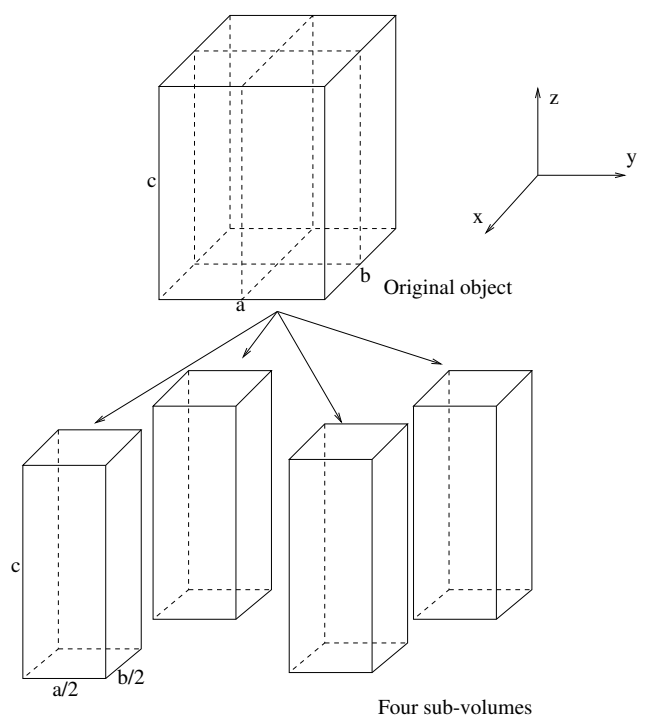

Fig. 3. Cone-beam decomposition. At each level, the volume is uniformly partitioned into four in the $x-y$ plane.

The recursion is continued until the cross sections of the 3D subimage in $x-y$ plane are of some desired minimum size $M_{\text {min }}$, and then the backprojections $\mathcal{B}_{M_{\text {min }},} P_{\text {min }}$ performed using weighted backprojection (3). That is, each point is still reconstructed using the Feldkamp algorithm, but with fewer cone projections. In the backprojection step, all the weighting parameters are still calculated in the original coordinates. The cone-beam projections are processed as stacks of fan-beam projections; therefore, the computational complexity is the same as performing $O(N)$ number of fast fan-beam backprojection, which is $O\left(N^{3} \log N\right)$, for an image of size $N \times N \times N$.

The computational cost of hierarchical backprojection, which is dominated by the radial shifting and angular filtering, is reduced by using short filters for these operations. To control the approximation error, it is useful to introduce oversampling of the data. An effective way to do so, is to perform one or more exact decompositions at the top of the hierarchy, where although the image size is reduced, the number of view angles is not. The data then becomes angularly oversampled. The number of exact decompositions is called the hold off factor. A holdoff of $Q=0$ corresponds to maximal angular decimation, whereas with $Q=1$ the first decomposition is exact, reducing the speedup by a factor of 2 but improving accuracy.

\section{NUMERICAL RESULTS}

We tested the fast Feldkamp algorithm on a $128 \times 128 \times 1283$ D Shepp-Logan head phantom The distance from the $\mathrm{x}$-ray source to the center of the 3-D image was 1.25 times the length of the lateral. The fan and cone-beam angles were 1.17 and 1.5 radians, respectively, which was barely enough to cover the whole object. We used a $375 \times 375$ detector plane and generated analytically cone-beam projections at 512 uniformly sampled source angles in 

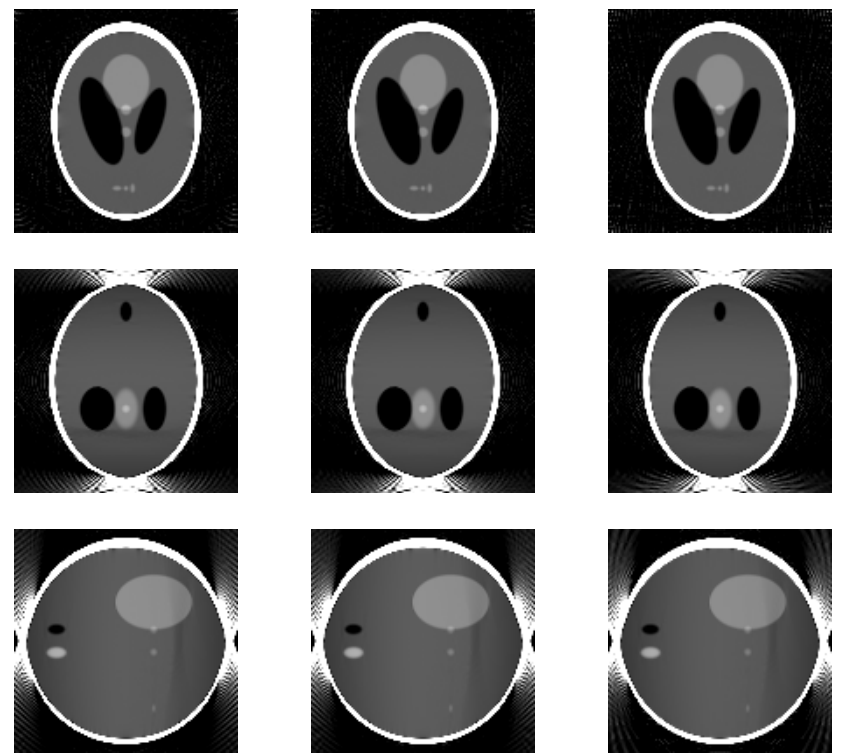

Fig. 4. Reconstruction results with equispaced planar detector. The three rows, from top to bottom, are $x y, y z$, and $x z$ slices. The left column is the standard Feldkamp reconstruction, whereas the middle and the right columns are the fast algorithm reconstructions with holdoff $Q=2$ and $Q=1$, respectively.

$[0,2 \pi]$. This resulted in a $512 \times 375 \times 375$ measurement data set. We performed experiments for both equispaced and equiangular detector geometries. The results for the equiangular geometry were essentially identical to those for the equispaced planar detector, and are not reported here.

Fig. 4 shows the cross sections of the 3-D phantom with respect to the $x, y$, and $z$ directions. Fig. 5 shows slices through reconstructed images of Fig. 4. The images display the artifacts typical of the single circle geometry and the Feldkamp algorithm for large values of $z$. However, importantly, the differences between the fast and conventional Feldkamp algorithms is very small. The fast algorithm with holdoff factor $Q=2$ is about three times faster then the conventional algorithm, with little if any degradation in image quality. Even with holdoff factor $Q=1$, the image quality is acceptable for some applications, with a 7 -fold speedup.

\section{REFERENCES}

[1] H. K. Tuy, "An inversion formula for cone-beam reconstruction,” SIAM J. Appl. Math., vol. 43, pp. 546-551, June 1983.

[2] L. A. Feldkamp, L. C. Davis, and J. W. Kress, "Practical conebeam algorithm," J. Opt. Soc. Am. A, vol. 1, pp. 612-619, June 1984.

[3] H. Turbell and P.-E. Danielsson, "Fast Feldkamp reconstruction," in International Meeting on Fully Three-Dimensional Image Reconstruction in Radiology and Nuclear Medicine, pp. 311-314, June 23-26 1999.

[4] H. Turbell, Cone-Beam Reconstruction Using Filtered Backprojection. $\mathrm{PhD}$ thesis, Linköping universitet, 2001.

[5] P.-E. Danielsson, "Iterative techniques for projection and
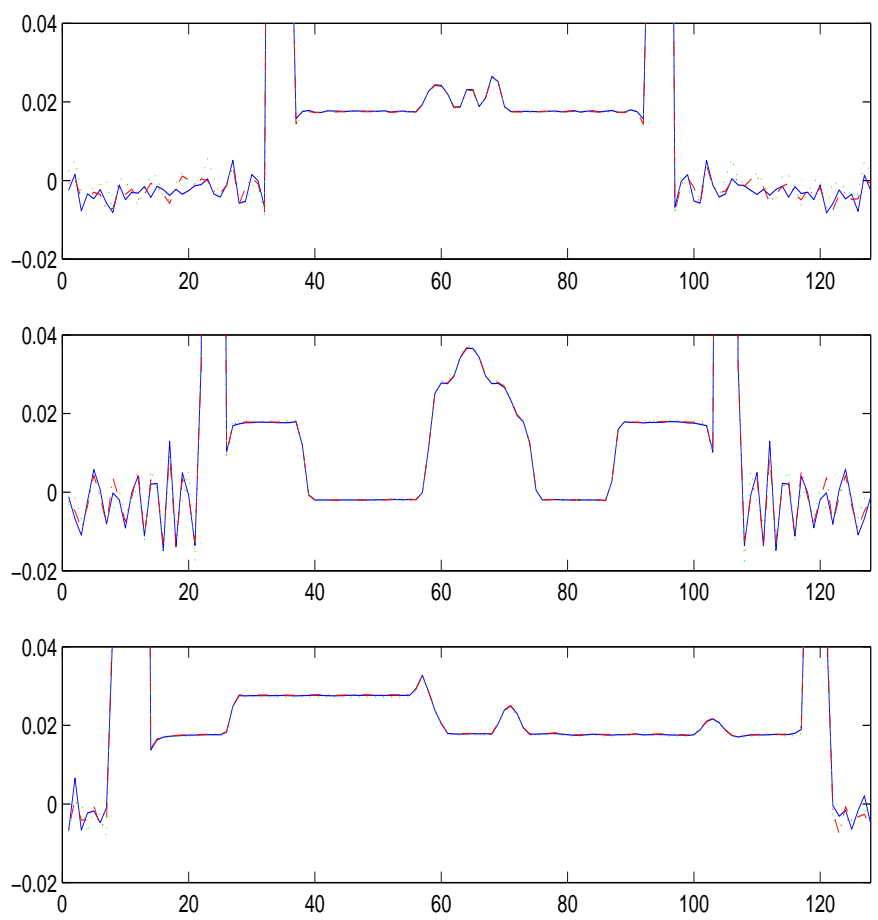

Fig. 5. Plots through images in Fig. 4. From top to bottom: 102-nd row of the top row images; 80 -th row of the middle row images; and 81-st column of the bottom row images. Standard Feldkamp reconstruction in solid line, and dashdot and dotted lines for the fast algorithm with holdoffs 2 , and 1 , respectively.

back-projection," tech. rep., Department of Electrical Engineering, Linköping universitet, 1997.

[6] S. Xiao, Y. Bresler, and D. C. Munson, Jr., " $\mathrm{O}\left(n^{2} \log n\right)$ native fan-beam tomographic reconstruction," in Proc. of IEEE International Symposium on Biomedical Imaging (ISBI), (Washington, D. C.), pp. 824-827, July 2002.

[7] F. Natterer and F. Wubbeling, Mathematical methods in Image Reconstruction. Philadelphia: SIAM, 2001. 